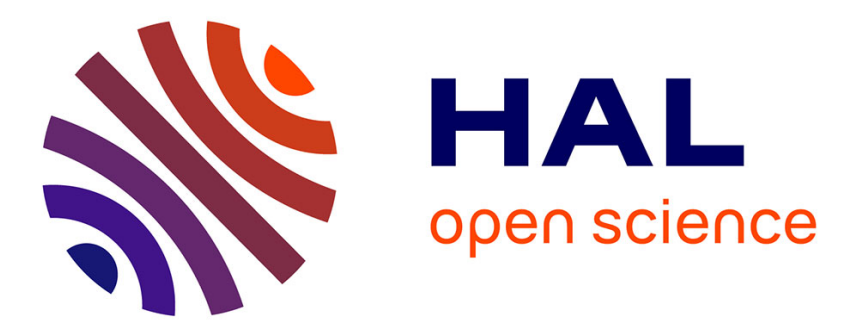

\title{
Observation of dissipative superluminous solitons in a Brillouin fiber ring laser
}

\author{
Eric Picholle, Carlos Montes, Claude Leycuras, Olivier Legrand, Jean \\ Botineau
}

\section{- To cite this version:}

Eric Picholle, Carlos Montes, Claude Leycuras, Olivier Legrand, Jean Botineau. Observation of dissipative superluminous solitons in a Brillouin fiber ring laser. Physical Review Letters, 1991, 66 (11), pp.1454. 10.1103/PhysRevLett.66.1454 . hal-01363621

\section{HAL Id: hal-01363621 \\ https://hal.science/hal-01363621}

Submitted on 10 Sep 2016

HAL is a multi-disciplinary open access archive for the deposit and dissemination of scientific research documents, whether they are published or not. The documents may come from teaching and research institutions in France or abroad, or from public or private research centers.
L'archive ouverte pluridisciplinaire HAL, est destinée au dépôt et à la diffusion de documents scientifiques de niveau recherche, publiés ou non, émanant des établissements d'enseignement et de recherche français ou étrangers, des laboratoires publics ou privés. 


\title{
Observation of Dissipative Superluminous Solitons in a Brillouin Fiber Ring Laser
}

\author{
Eric Picholle, Carlos Montes, Claude Leycuras, Olivier Legrand, and Jean Botineau \\ Laboratoire de Physique de la Matière Condensée, Université de Nice-Sophia Antipolis, \\ Parc Valrose, F-06034 Nice CEDEX, France \\ (Received 19 October 1990)
}

\begin{abstract}
Nonstationary stimulated Brillouin backscattering in a large-gain optical-fiber ring-cavity laser exhibits superluminous Stokes pulses of quasisoliton type and partial self-induced transparency for the pump. Experimental evidences, comforted by numerical simulation of the three-wave coherent model taking the acoustic-wave dynamics into account, show that this class of long transients occurs even with a cwcoupled pump wave.

PACS numbers: 42.65.Es, 42.50.Qg, 42.81.Dp
\end{abstract}

\begin{abstract}
Generation of large-scale coherent structures in a nonlinear optical medium caused by the action of parametric instabilities is one of the most interesting chapters in nonlinear optics. We report in this Letter the prediction and the first experimental observation of superluminous quasisolitons propagating along an optical fiber in a selfinduced transparency regime. Following our interpretation, this effect is related to the coherent behavior of the stimulated Brillouin backscattering (SBS) in a ring cavi-
\end{abstract} ty. ${ }^{1}$

SBS is the dominant stimulated scattering process in many optical media for long enough narrow-band laser waves. ${ }^{2,3}$ In the previous literature on optics, SBS is often described as a nonlinear susceptibility process by means of an intensity model. Through the Maxwell equations, such a model leads to a description of the interaction in which the phase dynamics is irrelevant. This approach is legitimate as long as the inertia of the acoustic wave can be put aside, assuming an adiabatic following of the latter due to its short spontaneous decay time. ${ }^{4}$

A more rigorous description of the spatiotemporal SBS behavior must account for the fact that SBS is a three-wave nonlinear interaction process which resonantly couples two electromagnetic waves and an acoustic wave through electrostriction. Thus, assuming slowly varying envelopes for the waves, neglecting optical dispersion, and respectively denoting the complex amplitude $E_{p}$ for the pump wave, $E_{S}$ for the counterpropagating Stokes wave, and $E_{a}$ for the acoustic wave, the three-wave equations read as follows in a coherent dimensionless form: ${ }^{4,5}$

$$
\begin{aligned}
& \left(\partial_{t}+\partial_{z}+\mu_{e}\right) E_{p}=-E_{S} E_{a}, \\
& \left(\partial_{t}-\partial_{x}+\mu_{e}\right) E_{S}=E_{p} E_{a}^{*}, \\
& \left(\partial_{t}+\mu_{a}\right) E_{a}=E_{p} E_{S}^{*},
\end{aligned}
$$

where the acoustic velocity has been neglected, due to its smallness relative to light velocity, and where $\mu_{e}\left(\mu_{a}\right)$ is the damping coefficient for the optical (acoustic) wave; note that in the limit $\partial_{t} E_{a} \ll \mu_{a} E_{a}$, this model yields the nonlinear susceptibility model. Evolution equations (1) recently allowed some new developments in the comprehension of nonstationary SBS in a ring-cavity fiberlaser experiment where a pulsed Stokes regime was stabilized by periodically modulating the $\mathrm{cw}$ pump beam with an intraring-cavity acousto-optic modulator (AOM). ${ }^{5}$ The experimental time scale for the Stokes pulse width $(=10 \mathrm{~ns})$ is comparable to the spontaneous acoustic decay time in silica $(\simeq 7 \mathrm{~ns}$ deduced from a spectral Brillouin bandwidth of $\Delta v=150 \mathrm{MHz}$ at a pump wavelength of $\left.\lambda_{p}=514.5 \mathrm{~nm}\right)^{6}$ and therefore absolutely calls for the use of the coherent description given by Eqs. (1). Moreover, additive terms in Eqs. (1) allow our numerical simulations to account for phase modulation due to the optical Kerr contribution. ${ }^{5}$

The parametric SBS instability associated with a cwlaser pump coupled into a bounded nonlinear optical medium of large enough SBS gain is responsible for the so-called Brillouin mirror: In the case of an ideal monochromatic cw pump wave and without any parasitic effect, the numerical treatment shows that, after some pulsed backscattered transients, the parametric SBS instability is saturated by the large depletion of the pump inside the medium and gives rise to its reflection into a backscattered cw Stokes wave. ${ }^{5}$ However, this ideal case does not correspond to the experiment; indeed, even the small reflectivity of the medium ends achieves a resonator. The influence of an external feedback on SBS temporal behavior in optical fibers has been investigated: ${ }^{7}$ When pump and Stokes waves are interacting in a cavity relaxation oscillations set in. This oscillatory regime superimposed to a strong continuum is described through the intensity model. However, very recent experiments show the generation of chaotic SBS in an optical fiber under cw pump conditions in the absence of external feedback. ${ }^{8}$ This dynamical behavior of the parametric SBS instability cannot be understood within the intensity model.

Here we are looking for a completely different timedependent regime for the Stokes pulse, namely, the superluminous quasisoliton regime, ${ }^{1}$ which involves the coherent dynamics of a self-similar backward- 


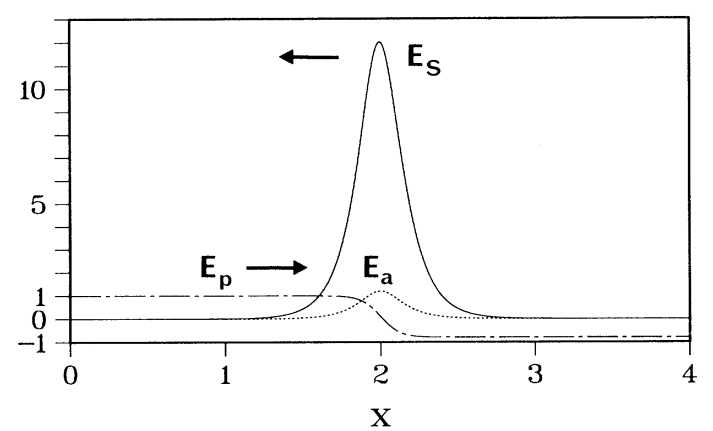

FIG. 1. Dissipative superluminous Brillouin soliton given by formulas (2), for $\mu_{a}=1$ and $\mu_{e}=10^{-2}$. Note that the pump is partially restored after the interaction.

propagating three-wave (pump-Stokes-acoustic) structure. For the three-wave interaction (1) in the nondissipative case (i.e., $\mu_{e}=\mu_{a}=0$ ), specific traveling-wave solutions have been studied in the forward-scattering case. 9,10 Corresponding solutions in the SBS case display new interesting features that persist in the dissipative case. One intriguing aspect of these solitonlike solutions is the superluminous velocity of the three-wave structure backward propagating along with the Stokes pulse. These solutions are special cases of a broader class of solitons previously described through an inverse scattering transform. ${ }^{11}$

However, in the case of silica optical fibers, it is impossible to neglect damping $\left(\mu_{i} \neq 0\right)$. Analytic superluminous traveling-wave solutions are still available if the pump attenuation is neglected, which is locally legitimate as long as $\mu_{e} / \mu_{a} \ll 1$; it is indeed the case in our experiment. Then particular solutions of system (1) in an infinite medium read (Fig. 1) as follows:

$$
\begin{aligned}
& E_{p}=P_{1}-P \tanh [(x+V t) / \Delta], \\
& E_{S}=S \operatorname{sech}[(x+V t) / \Delta], \\
& E_{a}=S P\left[2 /\left(S^{2}+P^{2}\right)\right]^{1 / 2} \operatorname{sech}[(x+V t) / \Delta],
\end{aligned}
$$

with the extra relationships

$$
P_{1}=\left(\mu_{e} \mu_{a}\right)^{1 / 2}, \quad S / P=\left(2 \mu_{a} / \mu_{e}-1\right)^{1 / 2},
$$

the velocity and the width of this backward complex structure being given by

$$
V=\frac{1}{1-\mu_{e} / \mu_{a}}, \quad \Delta=\frac{\left(\mu_{e} / \mu_{a}\right)^{1 / 2}}{P\left(1-\mu_{e} / \mu_{a}\right)} .
$$

Note that the value of the velocity is greater than unity (group velocity of light in the dimensionless equations we use). There is only one free parameter left, which, for practical reasons, will be taken as the pump amplitude at the far left end of the structure shown in Fig. 1, namely, $P_{1}+P=E_{p}(x+V t \ll-\Delta)=1$.

This superluminous self-similar Stokes pulse does not contradict by any means the special theory of relativity.

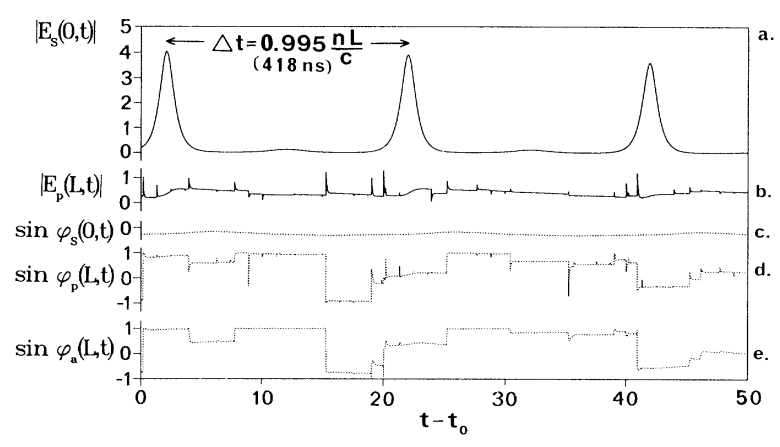

FIG. 2. Numerical computation of Eqs. (1) for the ring cavity with $\mu_{a}=10, \mu_{e}=10^{-2}$, and $L / \Lambda=20$, where $\Lambda$ is the SBS characteristic length (Ref. 5). Curve $a$, train of backscattered solitons [output Stokes component $E_{S}(0, t)$ ]; curve $b$, transmitted pump amplitude $E_{p}(L, t)$ showing spikes generated by random phase shifts; curve $c$, Stokes phase, almost insensitive to the pump phase jumps; curve $d$, strongly modulated pump phase; curve $e$, acoustic phase, following the pump phase jumps.

It rather can be viewed as the result of an amplification of the leading edges of the Stokes and acoustic pulses while, at the same time, their rears are depleted, the pump wave being partially restored after the interaction, in some cases with an opposite phase. This behavior is quite reminiscent of the self-induced transparency effect encountered in the coherent pulse propagation in a twolevel medium. ${ }^{12}$ No transportation of information can be obtained via this process which can only occur if a sufficiently extended background of Stokes light is available so that the superluminous self-similar amplification takes place. An additive interesting feature obtained by the numerical treatment of Eqs. (1) is that, when it is built, the Stokes pulse is no longer perturbed by pump phase fluctuations, which are compensated by the acoustic wave (Fig. 2).

The finite length of the fiber is a hindrance to achieve the asymptotic soliton behavior, but a kind of "infinite" medium may be partially recovered through a ring configuration in spite of the recoupling losses. In order to test this regime experimentally, we use as in Ref. 5 an $L=83 \mathrm{~m}$ optical single-mode silica fiber cavity pumped by the $\lambda_{p}=514.5 \mathrm{~nm}$ line of a single-mode argon-ion $\mathrm{cw}$ laser, the feedback being achieved by two mirrors (Fig. 3). This configuration allows the Stokes wave to spread

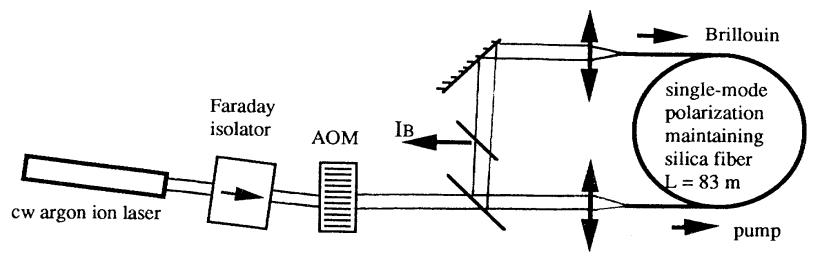

FIG. 3. Experimental setup. 
along the whole ring cavity ${ }^{13}$ and also a considerable lowering of the input pump power threshold since the Stokes wave is constantly counterpropagating with respect to the pump wave. Let us first describe the results obtained in a cw pump regime. When raising pump intensity above a threshold value of a few tens of milliwatts, two qualitatively different Stokes dynamical behaviors may be clearly distinguished.

(1) At low intensity (less than $100 \mathrm{~mW}$ coupled into the fiber), one observes trains of $\simeq 20 \mathrm{~ns}$ width pulses spaced at a fairly constant rate related to the round-trip flight time in the cavity $\Delta t_{L}=n L / c(\simeq 420 \mathrm{~ns})$, which means that there is only one pulse in the cavity. This regime is not very stable even though it may last several milliseconds. Still raising the pump intensity, two pulses or more may simultaneously be present inside the cavity.

(2) At higher intensity (beyond $250 \mathrm{~mW}$ ), a sudden change towards a $\mathrm{cw}$ regime (the Brillouin mirror) occurs, sometimes interrupted by a sequence of highamplitude modulations around the mean cw level.

Such wildly nonstationary behaviors are also obtained by numerical computation of Eqs. (1) in the ring configuration. Stochastic phase jumps for the pump at random time intervals around some coherence time $t_{\text {coh }}$ have been included in order to simulate the finite spectral bandwidth of the pump. One obtains a train of backscattered Stokes pulses $E_{S}(0, t)$ (cf. Fig. 2) for regime (1) when the narrow Stokes pulses, built from an initial small sound fluctuation, transform into the superluminous solitonlike pulses. Regime (2) was obtained for a higher pump input amplitude, and it is interesting to see that the random pump phase jumps, which also cause amplitude spikes for the pump, maintain a timedependent modulation regime over the $\mathrm{cw}$ level, and therefore prevent the steady Brillouin mirror regime in

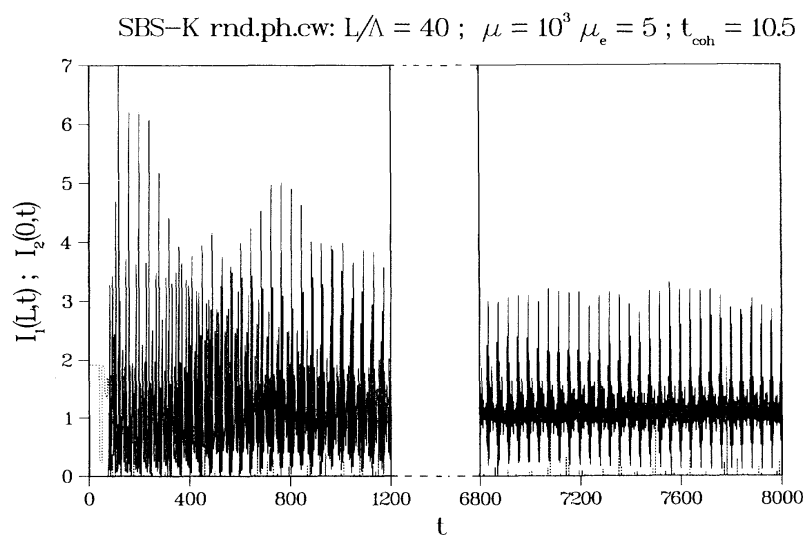

FIG. 4. Numerical computation of Eqs. (1) at high intensity level $\left(\mu_{a}=5, \mu_{e}=5 \times 10^{-3}\right.$, and $\left.L / \Lambda=40\right)$. The stochastic pump phase jumps are responsible for strong Stokes amplitude modulations around the Brillouin mirror cw level (exhibiting the main period $\left.\Delta t_{L}\right)$; here $t_{\mathrm{coh}} \simeq \Delta t_{L} / 4$. accordance with the experimental results (Fig. 4).

But working with a cw pump wave has a serious flaw since the input end of the fiber is permanently excited by the pump wave and the acoustic wave cannot delay by its own finite response time, therefore leading to the Brillouin mirror regime. Stabilization of the pulsed regime, even at high intensity level, may be obtained by periodically interrupting the pump wave with the AOM, during a time longer than the spontaneous acoustic damping time $\mu_{a}^{-1}$, as was done in Ref. 5. But here the modulation is set up outside the ring cavity, so that the Stokes pulses may develop their wings through the whole ring cavity. For a good efficiency, the modulation frequency must roughly correspond to $\left(\Delta t_{L}\right)^{-1}$ in the cavity. In this configuration the Brillouin mirror regime (steady or modulated) is totally prohibited and a pure solitonlike regime may be reached. We have obtained by this way sequences of well-shaped Stokes pulses for a few milliseconds. In Fig. 5(a) two groups of experimental Stokes pulses recorded at the output of the cavity during two consecutive sequences of qualitatively different dynamical behaviors are superimposed. The first one is associated with the building phase of Stokes fine evolutive structures separated by $420 \mathrm{~ns}$, which is, within a precision of $2 \mathrm{~ns}$, the round-trip flight time in the cavity at the group velocity of light in the fiber. The second one corresponds to a very stable sequence of hyperbolicsecant-like fitted pulses which repeat themselves every 414 ns. The stability of these solitonlike pulses is quite remarkable as can be verified in Fig. 5(b), which shows six superimposed uncorrelated groups of Stokes pulses. We also checked that a nonzero level of pump intensity is transmitted at the other end of the fiber. Superluminous velocity and partial self-induced transparency are certainly the most undisputable proofs of the physical

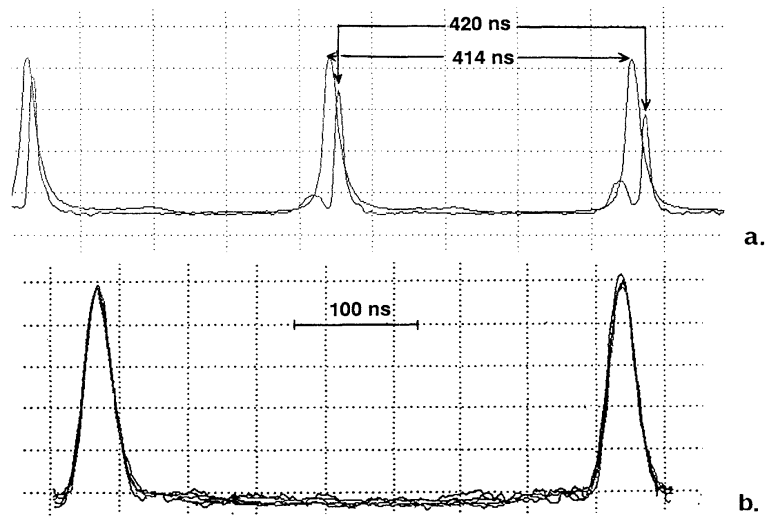

FIG. 5. (a) Experimental superluminous Brillouin Stokes solitons superimposed to the evolutive pulses spaced by $\Delta t_{L}$. (b) Experimental stability test of the Brillouin fiber-ring laser: six superimposed uncorrelated couples of pulses. 
relevance of the dissipative SBS quasisolitons. It is interesting to note that, by using such an externally modulated pump wave, a numerical treatment of Eqs. (1) shows the birth of the solitonlike profile by starting from completely stochastic amplitude and phase acoustic fluctuations.

It should nevertheless be kept in mind that what we observe is an incompleted growing quasisoliton. In fact, in the experiment a quasisoliton pulse is amplified and accelerated at each round trip in the cavity, but is also prevented from completing its growth every time it reaches the end of the fiber before being recoupled at the other end with a lower amplitude (approximately the half) due to losses pertaining to the ring setup.

In conclusion, it appears that dissipative superluminous quasisolitons can account for nonstationary dynamic behaviors observed for the first time in a Brillouin optical-fiber ring laser. Their contribution to the stabilization of the Stokes output was demonstrated as well as their physical relevance for a self-induced transparency effect on the transmitted pump wave.

The authors would like to thank $R$. Clapier and G. Sala for technical assistance. We also thank Dr. R. H. Stolen for the fiber used in the experiments, and the Centre National d'Etudes des Télécommunications (CNET) for financial support. Laboratoire de Physique de la Matière Condensée is URA No. 190 du CNRS.

'O. Legrand and C. Montes, J. Phys. (Paris), Colloq. 50, C3-147 (1989).

${ }^{2}$ E. P. Ippen and R. H. Stolen, Appl. Phys. Lett. 21, 539 (1972).

${ }^{3}$ D. Cotter, J. Opt. Commun. 4, 10 (1983).

${ }^{4}$ J. Coste and C. Montes, Phys. Rev. A 34, 3940 (1986).

${ }^{5}$ J. Botineau, C. Leycuras, C. Montes, and E. Picholle, J. Opt. Soc. Am. B 6, 300 (1989), and references therein.

$6 \mathrm{~J}$. Pelous and R. Vacher, Solid State Commun. 16, 279 (1975).

${ }^{7}$ I. Bar-Joseph, A. A. Friesem, E. Lichtman, and R. G. Waarts, J. Opt. Soc. Am. B 2, 1606 (1985).

${ }^{8}$ R. G. Harrison, J. S. Uppal, A. Johnstone, and J. V. Moloney, Phys. Rev. Lett. 65, 167 (1990).

${ }^{9}$ J. A. Armstrong, S. J. Sudhanshu, and N. S. Shiren, J. Quantum Electron. 6, 591 (1974).

${ }^{10} \mathrm{~K}$. Nozaki and T. T. Taniuti, J. Phys. Soc. Jpn. 34796 (1973); Y. Oshawa and K. Nozaki, J. Phys. Soc. Jpn. 36, 591 (1974).

${ }^{11}$ S. C. Chiu, J. Math. Phys. (N.Y.) 19, 168 (1978).

${ }^{12}$ S. L. McCall and E. L. Hahn, Phys. Rev. 183, 457 (1969).

${ }^{13}$ In Ref. 5 the intraring acousto-optic deflector cut the Stokes wings and prevented the formation of its hyperbolicsecant profile. 